\title{
Lambda Iteration and Genetic Algorithms Application to solve the Economic Load Dispatch Problem of Seven Nigerian Thermal Power Plants
}

\author{
Agber, Jonathan Uhaa and Anzaku Engla* \\ Department of Electrical and Electronics Engineering, Federal University of Agriculture, PMB 2373, Makurdi, \\ Benue State, Nigeria \\ * E-mail of the corresponding author: meetengi@gmail.com
}

\begin{abstract}
Economic load dispatch (ELD) is a method of earmarking the required load demand between the existing generations in power system and principally determining allocation of generators to each generation for various systems load levels. ELD is an essential component in power system planning and operation. ELD solutions are found by solving the conventional load flow equations, while at the same time ensuring that fuel costs are minimized. There are many methods developed for solving ELD problems, but this work is restricted to two, which are the Lambda Iteration Method (LIM) and the Genetic Algorithm (GA) modified with sorting algorithm. The main objective is to apply the two methods to solve ELD problems in power system networks by comparing the performance of GA method with that of LIM in terms of fuel cost efficiency. The usefulness of LIM and GA to solving economic dispatch problem is emphasized. Simulation results obtained on this network using GA and LIM verify their effectiveness in solving ELD problems. Lastly, GA and LIM approaches have been effectively applied to the harmonization of the Nigerian 32-bus system powered by seven thermal and three hydro generating units. The study shows that GA exhibits better results than LIM from both best possible generation allocations. The results obtained demonstrate that GA based method gave better solution in terms of fuel cost reduction, when compared with those obtained using LIM. By blending the probabilities of crossover and mutation, and the application of sorting techniques, computer usage time can be significantly reduced in the system with better fuel cost reduction.
\end{abstract}

Keywords: Economic load dispatch (ELD), genetic algorithm (GA), lambda iteration method (LIM), optimization, sorting algorithm.

DOI: $10.7176 / \mathrm{JETP} / 11-4-04$

Publication date: August $31^{\text {st }} 2021$

\section{Introduction}

The dimensions of the electric power systems are increasing significantly to sustain the energy requirements. A number of power plants are synchronized to supply the system load by interconnections of power stations. However, the rate at which power demand increases outweighs the rate of increase of generation, hence there is need for generators to be operated in an economic way (Wei-Tzer et-al. 2018).

The Economic Dispatch can be defined as the process of allocating generation levels to the generating units, so that the system load is supplied entirely and most economically. The aim of ELD is to reduce the overall cost of generation (Arunpreet et-al. 2014). The primary objective of economic dispatch is to reduce the total cost of generation at the same time keeping the operational constraints of the obtainable generation's resource and the management of generation systems to produce energy at the lowest possible cost to adequately satisfy end users, taking into consideration any operational limits of generation and transmission equipment.

Several methods have been implemented to solve the nonlinear objective function. The conventional methods such as Lambda Iteration method, Newton's method, Base Point and Participation factor method require the incremental cost curves to be continuous and monotonically increasing (Khamees, et-al. 2017). The solution of ELD using classical methods faces difficulties and has the possibility of producing local optimum results when dealing with non-convex cost functions.

Heuristic methods, like Genetic Algorithm, Evolutionary algorithm, Fuzzy logic and neural networks, have evolved to evade the above difficulties (Wei-Tzer et-al. 2018) and are known as complete and effective tools in power system optimization (Orike and Corne 2013). 
This study presents the combination of the Elitist and Sorting techniques to enhance the performance of Genetic Algorithm and application of LIM in solving economic load dispatch of the 32-bus system of Nigerian power system.

\section{Background of the Study}

During the past decade, efforts have been focused toward solving the Economic Dispatch problem, incorporating different kinds of constraints through the various mathematical programming and optimization techniques. Newton based algorithms have a problem in handling a large number of inequality constraints. Linear programming methods are fast and reliable, but the main disadvantage is associated with the piecewise linear cost approximation. Nonlinear programming methods have the difficulty of convergence and algorithm complexity (Khamees, et-al. 2017).

Abu (2015) proposed the economic load dispatch method with quadratic cost function by using Particle Swarm

Optimization (PSO) to investigate the Nigerian Grid System. The result showed that PSO method has the potential of finding optimums for both single and multiple objective functions, which can be either non-convex or discontinuous solution. The outcome showed that PSO was able to achieve the reduction of the cost of generating power.

Theoretically and practically, there are several mathematical methods to optimize the distribution of the generated power dispatching. The application of the artificial intelligence (Khamees, et-al. 2017) has proved its efficacy when applied to the optimization of objective functions.

Chiragkumar et al. (2016) solved Economic Load Dispatch problem in Power System using Genetic Algorithm method with the purpose of minimizing total fuel cost of generation. The data used was taken from the IEEE standard data sheet and GA method was implemented in MATLAB.

While Susheel et al. (2015) proposed an efficient optimization, technique based on genetic algorithm for solution of economic load dispatch problem with continuous and non-smooth cost function considering various constraints. The effectiveness of the proposed algorithm had been demonstrated on different systems with transmission loss in thermal power plant. Despite all these positive results, there still remains the need to test GA, blended with sorting algorithm, on real and existing power networks.

Bakare and Aliyu (2005) compared the performance of conventional GA (CGA) method to that of the micro-GA ( $\mu \mathrm{GA})$ method. The effectiveness of CGA and $\mu \mathrm{GA}$ to solving ED problem was verified on, 6-bus test system. Simulation results obtained on this network using both methods proved that the $\mu \mathrm{GA}$ displayed better results than the CGA from both fuel cost reduction and duration of computation.

The concept behind this study is to improve the economic load dispatch solution by merging insertion sorting algorithm with GA and making comparison with a very effective LIM method.

\section{Methodology}

\subsection{Fuel Cost Function}

The aspects of the cost categorized under dispatching procedures are the costs of fuel used in the fossil plants. The cost of fuel, labour, supplies and maintenance, all add up to give the total of cost operation. The relationship of fuel cost of each generator $\left(\mathrm{F}_{\mathrm{i}}\right)$ with the power output $\left(\mathrm{P}_{\mathrm{i}}\right)$ is given by a quadratic function

$$
F_{i}\left(P_{i}\right)=\sum_{i=1}^{N}\left(a_{i} P_{i}^{2}+b_{i} P_{i}+c_{i}\right) \quad N / h r
$$

where,

$F_{i}\left(P_{i}\right)$ : is the individual generation production in terms of its real power generation $\mathrm{P}_{\mathrm{i}}$,

$F_{i}=$ fuel cost of generator $i, P_{i}=$ power output of generator $i, a_{i}=$ measure of losses in the system, $b_{i}=$ represents the fuel cost, $\mathrm{c}_{\mathrm{i}}=$ includes salary and wages, interests and depreciation and is independent of generation and $\mathrm{N}=$ number of generation units.

The input of thermal plant is generally measured in Btu/hr and the power output is measured in MW. 


\subsubsection{Problem formulation}

The ELD problem is focused on reducing the total operating cost of a power system at the same time supplying the total load plus transmission losses within generator constraints of equality.

The objective of the ELD problem is to minimize the total fuel cost of thermal plants subject to the constraint of equality in real power balance.

$$
\sum_{i=1}^{W} P_{i}-P_{L}-P_{B}=0
$$

And the limits of the generation outputs

$$
P_{\mathrm{i}(\min )} \leq P_{\mathrm{i}} \leq P_{\mathrm{i}(\max )}
$$

where:

$\mathrm{P}_{\mathrm{D}}$ is the total current system load demand and $\mathrm{P}_{\mathrm{L}}$ is the total system transmission losses.

The thermal plant can be expressed as input-output models (cost function), where the input is the fuel cost and the output is the power generation of each unit. In practice, the cost function could be represented by a quadratic function.

$$
F_{\mathrm{i}}\left(P_{\mathrm{i}}\right)=A_{\mathrm{i}} \times P_{\mathrm{i}}^{2}+B_{\mathrm{i}} \times P_{\mathrm{i}}+C_{\mathrm{i}}
$$

The incremental cost curve data are obtained by taking the derivative of the unit input-output equation resulting in the following equation for each generator:

$$
\partial F_{i}\left(P_{\mathrm{i}}\right) / \partial P_{\mathrm{i}}-2 A_{\mathrm{i}} \times P_{\mathrm{i}}+B_{\mathrm{i}}
$$

Solving the ELD equations for a specified system requires an iterative approach since all unit generation allocations are embedded in the equation for each unit. Transmission losses can be calculated directly using the approximate method of expression through the use of B-coefficients. This method (TCN 2012) relies on the notion that under normal operating condition, the transmission loss is quadratic in the injected bus real power. The $\mathrm{B}_{\mathrm{mn}}$ matrix loss formula is, therefore, given as

$$
P_{L}=\sum_{i=1}^{N} \sum_{j=1}^{N} P_{i j} B_{i j} P_{j}+\sum B_{0 i} P_{i}+B_{00} \quad \text { MW }
$$

where,

$\mathrm{B}_{\mathrm{ij}}$ are coefficients, constants for certain conditions, voltage and angle of load buses and $\mathrm{P}_{\mathrm{i}}, \mathrm{P}_{\mathrm{j}}$ are real power injection at the ith and jth buses.

Using Langrange multiplier, an augment's function is given as

$$
L\left(F_{i}, \lambda\right)=F\left(P_{i}\right)+\lambda\left(P_{D}-P_{L}-\sum_{i=1}^{N} P_{i}\right)
$$

where, $\lambda$ is the Lagrangian multiplier.

Necessary conditions (TCN 2012) for the optimization problem are

$$
\frac{\partial\left(F_{i} \lambda\right)}{\partial P_{i}}=\frac{\partial F\left(P_{i}\right)}{\partial P_{i}}+\lambda\left(\frac{\partial P_{i}}{\partial P_{i}}-1\right)=0, \quad(\mathrm{i}=1,2,3, \ldots . . \mathrm{N})
$$

Solving equation (8) gives

$$
\frac{\partial F\left(P_{i j}\right)}{\partial p_{i}}=\lambda\left(1-\frac{\partial p_{L}}{\partial p_{i}}\right)
$$

For incremental cost of the $\mathrm{i}^{\text {th }}$ equation (9) becomes

$$
\frac{\partial\left[F_{i} D\right.}{\partial P_{i}}=\lambda_{i}\left(1-\frac{\partial P_{L}}{\partial P_{i}}\right), \quad(\mathrm{i}=1,2,3, \ldots . . \mathrm{N})
$$


Where,

$\frac{\partial\left[F_{i} j\right)}{\partial P_{i}}$ is the incremental cost of the $i^{\text {th }}$ generator $(\mathrm{N} / \mathrm{MWh})$

$\frac{\partial P_{R}}{\partial P_{i}}$ is the incremental transmission losses.

Equation (8) represents the correct optimization equation (Wei-Tzer et-al. 2018) and

$$
\frac{\partial\left[F_{i} D\right.}{\partial P_{i}}=P_{D}+P_{L}-\sum_{i=1}^{Y} P_{i}=0
$$

By differentiating the transmission loss equation (6) with respect to $\mathrm{P}_{\mathrm{i}}$, the incremental transmission loss can be obtained as

$$
\frac{\partial P_{L}}{\partial P_{i}}=\sum_{j=1}^{N} 2 B_{i j} P_{j}
$$

And by differentiating the cost function of equation (1) with respect to $\mathrm{P}_{\mathrm{i}}$, the incremental cost can be obtained as

$$
\frac{\partial F\left(P_{i}\right)}{\partial p_{i}}=2 a_{i} P_{i}+b_{i}, \quad(\mathrm{i}=1,2,3, \ldots . . \mathrm{N})
$$

And the solution

$$
2 a_{i} P_{i}+b_{i}=\lambda\left(1-2 \sum_{i=1}^{N} B_{i j} P_{i}\right), \quad(\mathrm{i}=1,2,3, \ldots . \mathrm{N})
$$

Fine tuning equation (14), the expression for obtaining $\mathrm{P}_{\mathrm{i}}$ can be obtained as:

$$
2 a_{\mathrm{i}} P_{\mathrm{i}}+b_{\mathrm{i}}=\lambda\left(1-2 b_{\mathrm{ij}} P_{\mathrm{i}}-2 \sum_{\mathrm{j}=1}^{\mathrm{N}} B_{\mathrm{ij}} P_{\mathrm{j}}\right), \quad(\mathrm{i}=1,2,3, \ldots \mathrm{N})
$$

Equation (15) provides a useful expression for obtaining the values of $\mathrm{P}_{\mathrm{i}}$ for range of $\lambda$ values with the aid of an appropriate numerical method or software algorithm.

\subsection{Lambda Iteration Method}

The lambda-iteration is a very popular method for solving economic load dispatch problems. Lambda-iteration is the method by which the optimum lambda value that leads to satisfaction of the system power balance constraints is determined. Using the above economic interpretation of $\lambda$, whenever the choice of $\lambda$ is such that it leads to insufficient generation, $\lambda$ must be increased so as to make it useful for the generators to increase production. Anytime an increase in generation is experienced, $\lambda$ must be decreased to limit generation (Chiragkumar et al. 2016). Using this method, the first step is to calculate the minimum and maximum lambda for each generating unit

$$
\begin{aligned}
& \lambda_{\min (N)}=\frac{d F_{i}\left(p_{i \mid \min j_{j}}\right)}{d p_{i}} \\
& \lambda_{\max (N)}-\frac{d F\left(p_{i(\max )_{j}}\right)}{d p_{i}}
\end{aligned}
$$

The starting value of lambda can be obtained from

$$
\lambda=\frac{2 \max +\lambda_{\min }}{2}
$$

At the point, where lambda obtained produces inadequate generation, the minimum value is updated to $\lambda_{\min }=\lambda$. If excess generation is obtained, the maximum lambda value is updated to $\lambda_{\max }=\lambda$. The new iteration value using ( $\left.\lambda_{\min }, \lambda_{\max }\right)$, is then applied to equation (18) until the lambda value that leads to the satisfaction of the power balance constraint is obtained. The step by step procedure of for implementation of lambda iteration algorithm in Matlab is given by a flowchart in Figure 1. 


\subsection{Genetic Algorithm}

GA is an adaptive search technique that acquires its model from the genetic process of biological organisms centered on evolutionary theory. The main advantage is that GA uses only the induced information and hence independent of the nature of the search space such as smoothness and convexity. The GA is more likely to converge toward the global solution, because it simultaneously evaluates many points in the parameter space.

\subsubsection{Formation of genetic algorithm}

The GA are computational models and are potent probabilistic search algorithms based on the procedure of natural selection and natural genetics. GA works with a population of binary strings by searching several likely solutions at the same time. By employing genetic operators, they switch information between the likely solutions, hence reducing the possibility of ending at a local optimum. The genetic model of computation can be implemented by having arrays of bits or character to represent the chromosomes. Simple bit manipulation operations aid the implementation of crossover, mutation and other operations.

A single iteration is referred to as a generation. The first generation is a population of randomly generated individuals. Premature convergence is one of the major difficulties with GA and most search algorithm. It has been observed that this problem is closely tied to the problem of losing diversity in the population. To avoid premature convergence, GA parameters should be meticulously chosen.

\subsubsection{ELD solution through GA}

The algorithm starts from an initial population generated randomly. This population undergoes three genetic operations, selection, crossover and mutation to produce a new generation after duly considering the fitness of strings, which corresponds to the objective function for the concerned problem. A trial solution for the problem requires the selection of a number of populations for a generation and a number for several such generations in order to find the best fitness of strings in that trial. Several such trails are considered to evaluate the overall best objective function. The best value of the fitness of the strings is dependent on the number of populations in a generation, the number of generations and the number of the trials, while solving the problem.

\subsubsection{Implementing insertion sort algorithm on initial population}

A sorting algorithm is an algorithm that puts items of a list in a definite order. The most-used arrays are numerical order and lexicographical order. Effective sorting is useful for optimizing the use of other algorithms that require sorted lists to work correctly. This work employed the use of insertion sort algorithm to fine-tune the initial population generated in GA in order to enhance speed and accuracy of the result obtained.

\subsubsection{Genetic operations:}

\section{A) Fitness evaluation}

The fitness function is derived from the objective function and used to determine the suitability of the solution from the initial set of solution of the problem.

Fitness function (Chiragkumar et al. 2016) is given as

$$
f(x)=\frac{1}{1+F(x)}
$$

Where,

$\mathrm{F}(\mathrm{x})$ is the objective function and $\mathrm{f}(\mathrm{x})$ is the fitness function

\section{B) Selection with embedded elitism}

Selection creates a new population from the old population. In selection process, strings are picked up from the present population based on their fitness values to form a new population. For this process, Roulette Wheel selection procedure is followed. Elitism is based on a comparison of the augmented costs for the 
strings. The basic premise of elitism is survival of the fittest.

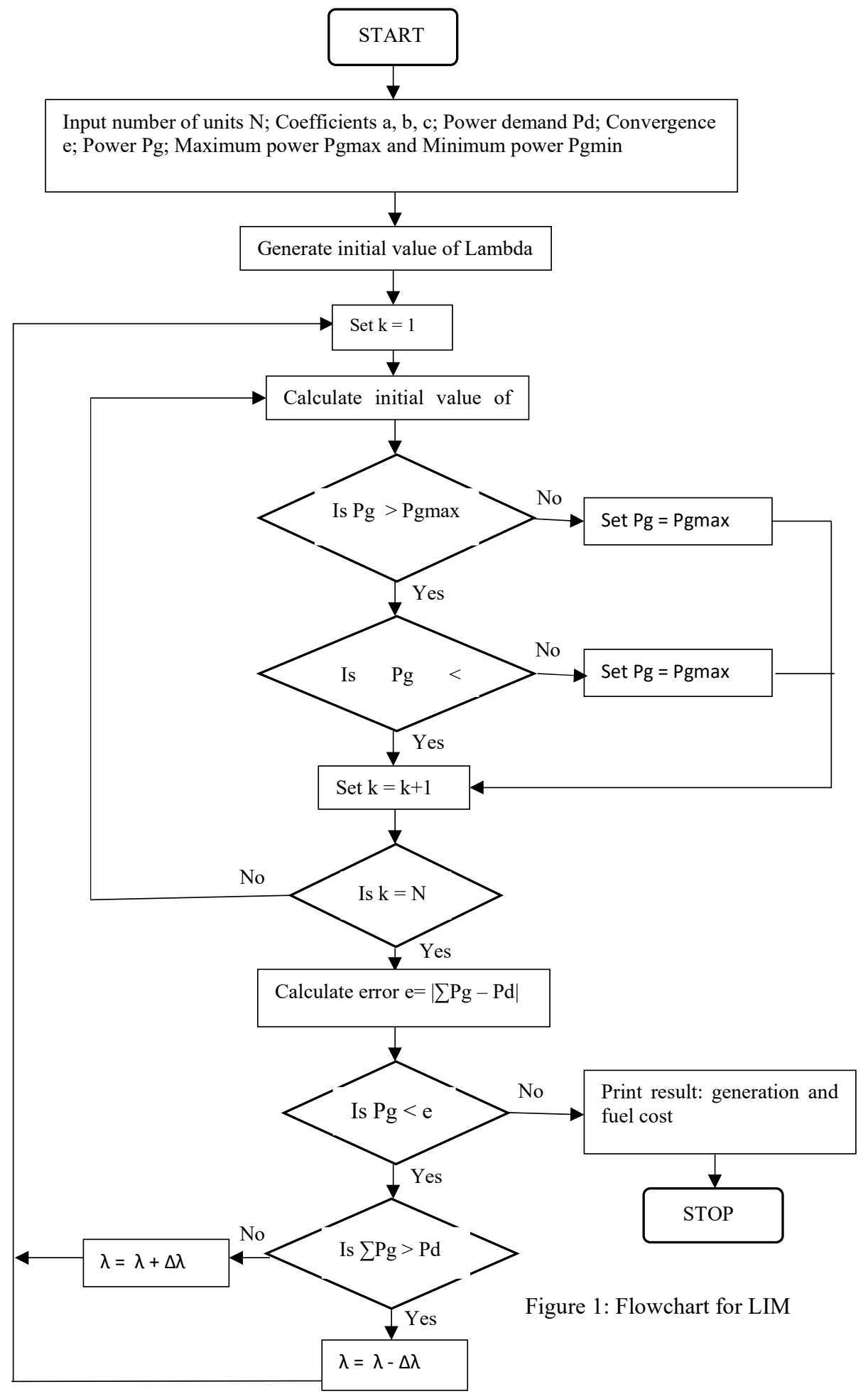

\subsubsection{Implementation of GA solution with line flow constraints}

The various stages involved in the solution algorithm for GA are as follow: 
I). Select the appropriate GA probabilities, population size, number of generations, sub-strings length and the number of trials.

II). Generate initially randomly coded strings of values as population members in the first generation and perform sorting on the population.

III). Decipher the population to get power generation of the units in the strings.

IV). Perform load flow taking into consideration the unit generation's in step (III) excluding that of the slack bus, in order to estimate the system transmission losses and slack bus generation.

V). Evaluate the fitness of population members.

VI). Execute selection based on reproduction considering Roulette Wheel procedure with embedded elitism followed by crossover with embedded mutation will create the new population for the next generation.

Steps (II) to (VI) are repeated for all the number of generations and the minimum augmented cost is noted for the first trial. This operation is carried out for the selected number of trials and the overall minimum for the augmented cost is taken as the solution point.

\subsubsection{Genetic algorithm for ELD}

The step by step procedure for implementation of Genetic algorithm in Matlab is given by the flowchart in Figure 2 .

\subsection{Results and Discussion}

The result of the ELD system after the enhancement of genetic algorithm with insertion sort algorithm are discussed and compared with classical LIM. The algorithms were implemented using MATLAB to solve ELD problem. The main goal is to minimize the cost of generation of thermal plants using the modified GA, and classical LIM. The performance was evaluated with losses for two set of generator data, which were categorized as Problem A.

\subsection{Problem A: Nigerian Power System Network}

The test has been performed on the standard model of the 32 bus Nigerian power network consisting of ten power generating systems, of which four are thermal plants while three are hydro power systems. The typical power demand is considered to be $2847 \mathrm{MW}$. The coefficients of fuel cost are given in Tables 1 from PHCN National Control Center Oshogbo daily operational report for May, 2012 (TCN 2012). Maximum and minimum power limits are also given in Table 1. Figure 3 shows the single line diagram of the 32 bus $330 \mathrm{kV}$ Nigerian power grid system.

Table 1 Cost coefficients for the four Nigerian thermal power plants

\begin{tabular}{|c|c|c|c|c|c|}
\hline Unit number & $\mathrm{A}_{\mathrm{i}}$ & $\mathrm{B}_{\mathrm{i}}$ & $\mathrm{C}_{\mathrm{i}}$ & $\begin{array}{c}\text { Minimum } \\
\text { power limit }\end{array}$ & $\begin{array}{l}\text { Maximum } \\
\text { power limit }\end{array}$ \\
\hline Sapele & 0.1366 & -8.3320 & 471542.6515 & 137.5 & 550.0 \\
\hline Delta & 1.1222 & 166.2918 & 56900.0956 & 75.0 & 375.0 \\
\hline Afam & 0.1527 & 92.9600 & 3316.6800 & 135.0 & 540.0 \\
\hline Egbin & 0.0310 & 13.1000 & 12787.0000 & 275.0 & 1100.0 \\
\hline Olorunshogo & 0.0322 & 10.4230 & 3377.0612 & 70 & 335 \\
\hline Omotosho & 2.5969 & 841.4102 & 479996.7373 & 75 & 335 \\
\hline Geregu & 0.001127 & 117.1114 & 24439.5109 & 138 & 414 \\
\hline
\end{tabular}

Source: Transmission Company of Nigeria Oshogbo Annual Report, (TCN, 2012).

Parameters for Genetic Algorithm:

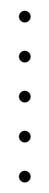

Parameters for Lambda Iteration Method:

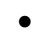

$\bullet$
Length of the sting $=64$

Population of string, pop $=800$

Crossover probability, $\mathrm{Pc}=0.867$

Mutation probability, $\mathrm{Pm}=0.01$

Elitism value $=0.15$

Maximum iteration $=1000$

Convergence $=0.01$ 


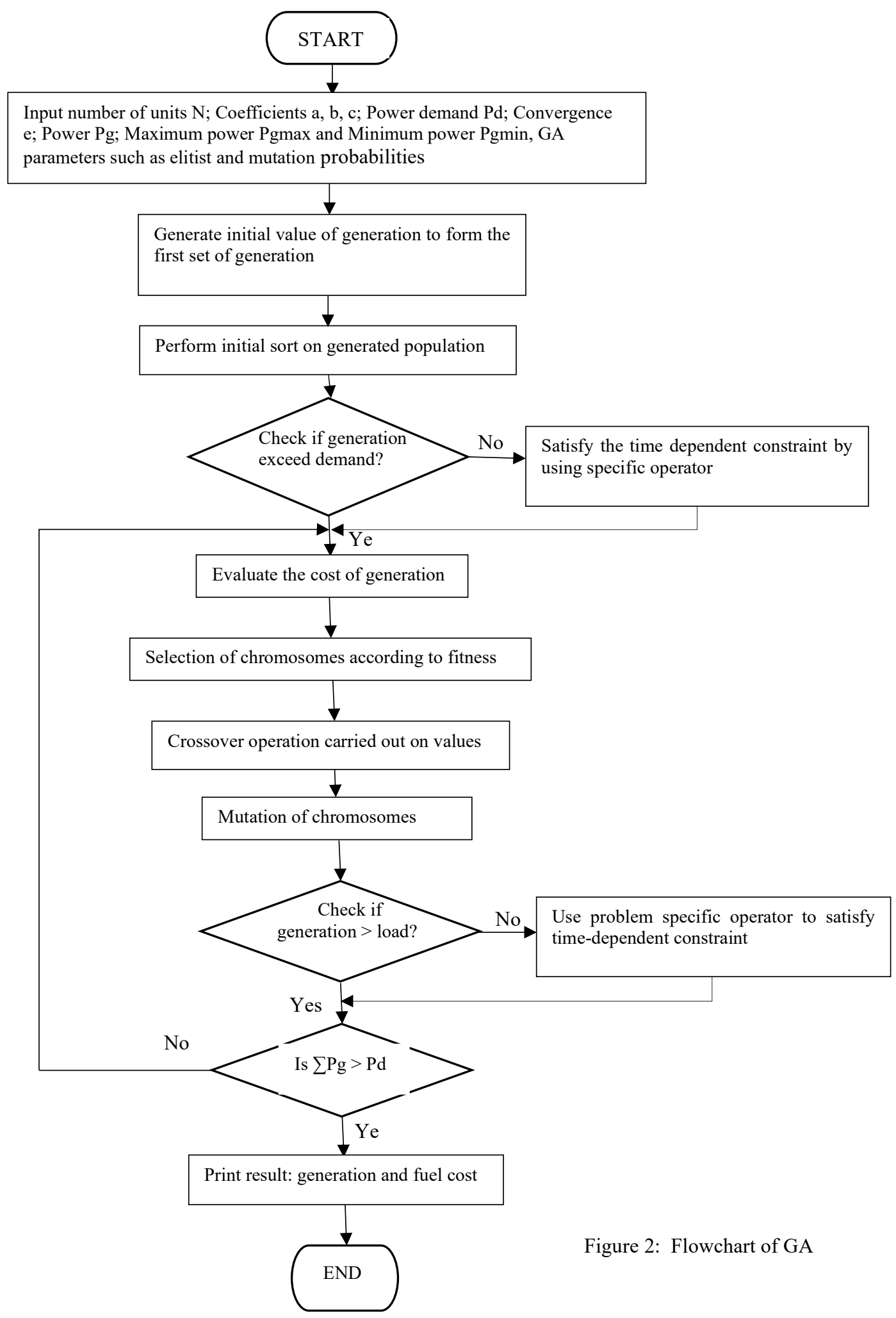




\subsection{Optimum solution using GA and LIM for problem A}

The simulated program gives the generated power required, the total cost, total losses and the error. The simulated results for lambda iteration method are shown in Table 2. The GA and LIM techniques were then used to evaluate the thermal units with the transmission losses parameters included. The results achieved are as shown in Table 2 for the two methods. GA once more offers superior outcome with a reduction of about $\$ 5910$ per hour when compared with the results obtained through LIM. However, GA required more computational time compared with LIM. The total production costs obtained via GA approach are also lower when compared with the values obtained using the LIM approach. GA yielded much smaller network losses when compared with LIM method.

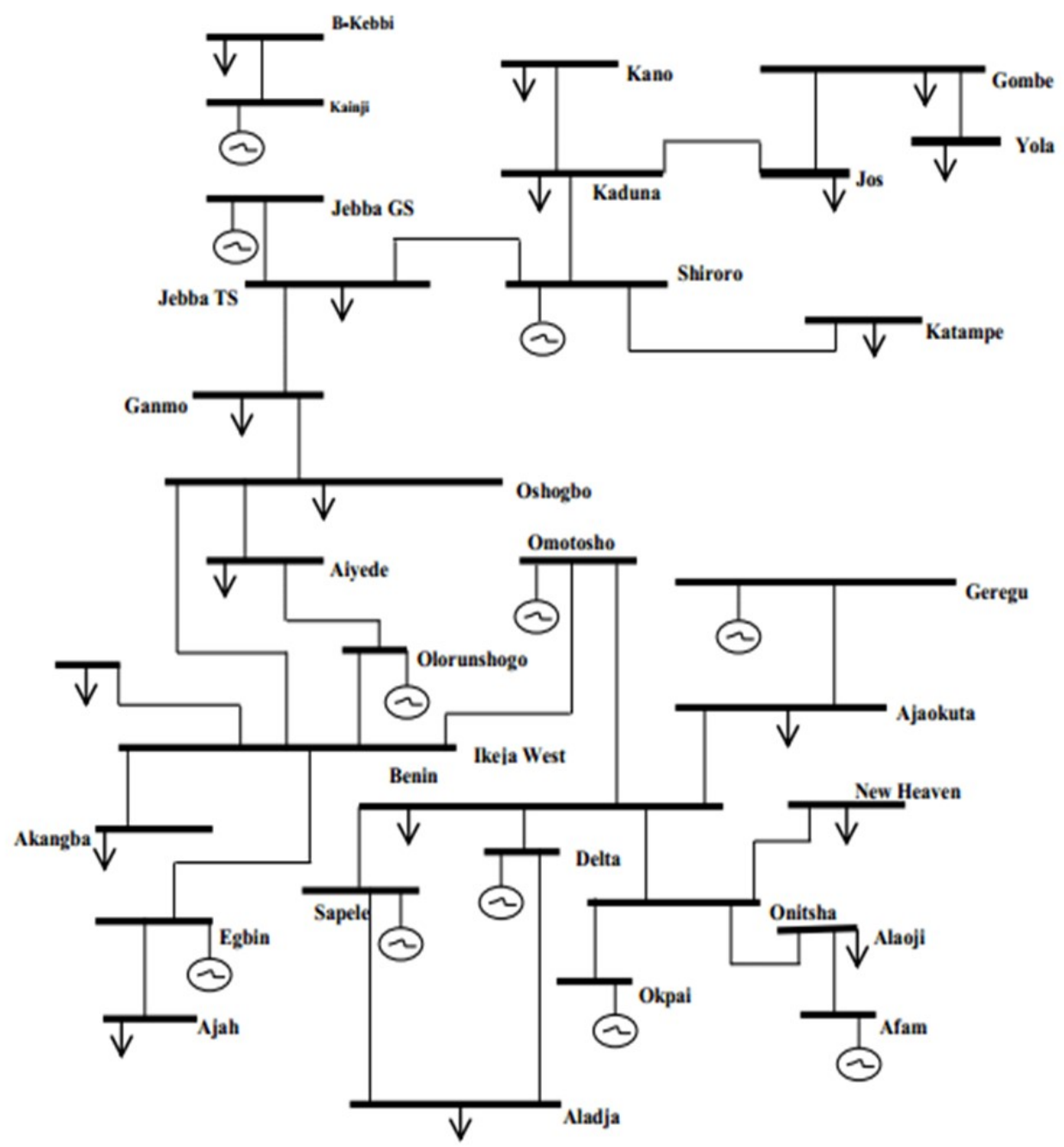

Figure 3: 32-bus Nigerian power system for problem A 
Table 2 Results obtained using GA and LIM for Problem C

\begin{tabular}{|l|l|l|}
\hline \multirow{2}{*}{ Power Plant/Parameters } & \multicolumn{2}{c}{ Method } \\
\cline { 2 - 3 } & & GA LIM \\
\hline Sapele & 467.697 & 313.539 \\
\hline Delta & 75 & 75 \\
\hline Afam & 135 & 135 \\
\hline Egbin & 1100 & 1037.57 \\
\hline Olorunshogo & 335 & 335 \\
\hline Omotosho & 75 & 75 \\
\hline Geregu & 190.184 & 414 \\
\hline Shiroro & 390.21 & 390.21 \\
\hline Kainji & 415.55 & 415.55 \\
\hline Jebba & 431.83 & 431.83 \\
\hline Total power generated (MW) & 3613.57 & 3622.80 \\
\hline Total network losses (MW) & 16.4192 & 25.6455 \\
\hline Total power demand (MW) & 2359.56 & 2359.56 \\
\hline Total cost (N/hr) & 1271520 & 1277430 \\
\hline Error & 0.00717521 & 0.0995033 \\
\hline Processing time (sec.) & 50.7982 & 0.705273 \\
\hline
\end{tabular}

\section{Conclusion}

Application of GA and LIM methods to ELD has been explored and tested for total fuel cost saving. The results obtained using Matlab show that a simple genetic algorithm can give good result, when genetic operations, such as sorting, balanced reproduction; simple mutation and crossover in binary codes are employed. GA is applicable in a large-scale system where the numbers of constraints are very large and the computer processing time is not a big factor.

In these research GA was further enhanced with the used of sorting technique to improve the result by the program developed in Matlab. The developed system was then tested and validated on the 32-bus Nigerian power network. The results obtained by the use of GA were compared against the result obtained by LIM method. The total fuel cost obtained by GA method was lower than that obtained by LIM, regardless of the differences in the error and loss values obtained for both methods. Solutions obtained with the developed GA economic dispatch program were better than those obtained by LIM method especially in terms of cost of fuel, although LIM solution was faster (used less processing time). Hence GA produced a better fuel reduction than LIM.

\section{REFERENCES}

Abu, U.A. (2015). Power System Economic Load Dispatch Using Particle Swarm Optimization. International Journal of Advanced Engineering Research and Technology (IJAERT), 3, 202-205.

Arunpreet, K., Harinder, P., Singh, A. B. (2014). Analysis of Economic Load Dispatch Using Genetic Algorithm, International Journal of Application or Innovation in Engineering \& Management, Volume 3, Issue 3.

Bakare, G.A., Aliyu, U.O., Venayagamoorthy G.K., Shua'bu, Y,A. (2005). Genetic Algorithms Based Economic Dispatch with Application to Coordination of Nigerian Thermal Power plants. IEEE Power society General Meeting. http://ieeexplore.ieee.org

Chiragkumar, P., Rakesh, B.S., Minaxi, L. P., and Rajesh, D.P. (2016). An Analysis of Economic Load Dispatch in Power System Using Genetic Algorithm. International Journal of Advanced Research in Electrical, Vol. 5, Issue 1.

Khamees, A. K., El-Rafei, A., Badra, N. M. and Abdelaziz, A. Y. (2017). Solution of optimal power flow using evolutionary-based algorithms. International Journal of Engineering, Science and Technology, 9, 55-68

Orike, S and Corne, W.D. (2013). Constrained Elitist Genetic Algorithm for Economic Load Dispatch: Case Study on Nigerian Power System. International Journal of Computer Applications, Volume 76- No.5.

Susheel, K. D., Achala, J. and Huddar, A.P. (2015). A Traditional Approach to Solve Economic Load Dispatch Problem Considering the Generator Constraints. IOSR Journal of Electrical and Electronics Engineering (IOSR-JEEE), 10, 27-32.

Transmission Company of Nigerian (TCN) (2012) National Control Centre Oshogbo, Generation and Transmission Grid Operations Annual Technical Report for 2012, TCN publisher.

Wei-Tzer, H., Kai-Chao, Y., Ming-Ku, C, Feng-Ying, W., Cang-Hui, Z., Yung-Ruei, C., Yih-Der, L. and YuanHsiang, H. (2018). Derivation and Application of a New Transmission Loss Formula for Power System Economic Dispatch. Energies, 11, 417 